\title{
Two Dimensional Laser Galvanometer Scanning Technology for Additive Manufacturing
}

\author{
Huang Jigang, Qin Qin, Wang Jie, and Fang Hui
}

\begin{abstract}
In this paper, the barrel-pincushion distortion of the two dimensional laser galvanometer scanning system is analyzed, and investigates the performance of conic fitting algorithm for galvanometer scanning based additive manufacturing (AM) to correct the distortion. Moreover, a scanner delay strategy of galvanometer scanning technology for $A M$ is proposed to improve the geometric precision and surface finish. The proposed scanner delay includes four kinds of delays, which are laser on-off delay, jump delay, mark delay and corner delay. While the effects of these delays are also revealed. To validate the performance of the conic fitting algorithm and the proposed scanner strategy for AM, a stereolithography (SLA) based 3D printer with galvanometer scanning technology is designed and the experiment is conducted on the 3D printer. The results show that the conic fitting algorithm has a contribution to compensate the barrel-pincushion distortion. The results also show that the scanner delay strategy can avoid the excessive material accumulation effectively and correct the contour error which caused by the non-uniform laser power density as well as the inaccurate scanning movement. The work in this paper indicates that the conic fitting algorithm and the scanner delay strategy are effective to improve the geometric precision and surface finish of parts fabricated by AM with galvanometer scanning technology.
\end{abstract}

Index Terms-Barrel-pincushion, galvanometer, scanner delay, SLA.

\section{INTRODUCTION}

Since the first technique for additive manufacturing (AM) became available for fabricating prototypes and models in the late 1980s. AM technology has received much attention and is presently one of the rapidly developing advanced manufacturing techniques in the world. AM techniques, which also referred to as 3D printing, differs from conventional manufacturing techniques such as subtractive manufacturing techniques (i.e., turning, grinding or milling), and formative manufacturing techniques, $3 \mathrm{D}$ printing techniques are applied for producing physical objects layer by layer from CAD models with a short period [1]. While the advantage of fabricating some complex, irregular shaped parts, which cannot be easily produced by the conventional manufacturing techniques, making 3D printing a prominent manufacturing technique with great commercial potential. Now, types of 3D printing techniques have already been used in many fields, such as model fabrication, materials, aerospace, biomedical and Micro/Nano manufacturing, and

Manuscript received May 2, 2018; revised July 19, 2018.

The authors are with School of Manufacturing Science and Engineering, Sichuan University, Chengdu, China (e-mail: hjigang@foxmail.com). always showed satisfactory performance [2].

Laser galvanometer scanning system is applied more and more widely to the $3 \mathrm{D}$ printing techniques, including selective laser melting, stereolithography (SLA), and selective laser sintering (SLM). As the development of galvanometer based scanning technology and its scanning controller, the 3D printing techniques with laser galvanometer scanning system are expected to achieve high precision and efficiency [3]. However, distortion caused by laser galvanometer scanning, especially the nonlinear distortion will decrease the precision greatly [4]. Moreover, the inharmonious between laser beam and motion system also can result to geometric error. The non-uniform laser power density on the material can affect the surface finish of parts which are fabricate by laser galvanometer based 3D printing techniques [5].

Some studies have been taken to research on these factors to improve the precision of laser galvanometer scanning based 3D printing techniques. Xi Luo et al. (2017) [6] demonstrated two control techniques to deliver uniform laser density on the material to be processed: a control algorithm to optimize the scanning trajectory according to the scanner's dynamic characteristics as well as the job accuracy requirements, and a method to modulate laser based on scanner speed change. Xi Luo et al. also analyzed the performance of these two control methods respectively. GuangY Li et al. (2014) [7] analyzed the barrel and pincushion distortion in laser galvanometer scanning system, and presented a graphic correction algorithm. The experiments showed that the algorithm provided satisfactory correction effect for the laser galvanometer in 3D printing techniques. Zhao Qun, Wang Chao et al. (2012) [8] also analyzed the error in laser galvanometer scanning system, and discussed three correction methods, which are conic fitting algorithm, geometric correction algorithm based on plane coordinate transformation and angle compensation correction method. Han Woong Yoo, Shingo Ito et al. (2016) [9] proposed the iterative learning control for a galvanometer scanner to achieve high speed, linear, and accurate bidirectional scanning for scanning laser microscopy. The experimental results verify the benefits of iterative learning control of its wide control bandwidth, enabling a faster, more linear, and more accurate scanning without a phase lag and a gain mismatch.

Now, the distortion of laser galvanometer scanning for 3D printing has been studied, while the experimental verification is still limited. Moreover, the scanner delay strategy for laser galvanometer scanning based 3D printing has rarely been studied before. Considering the scanner delay strategy has contribution to uniform laser power density on the material, which relates to the geometric 
precision and surface finish of the parts closely, it is worthy and necessary to be further studied. In this paper, the barrel and pincushion distortion were analyzed, and the performance of conic fitting algorithm was validated by experiments. A scanner delay strategy was also proposed and the validity was verified in the paper. The paper is organized as follows: the barrel and pincushion distortion are discussed in Section II, Section III introduces the conic fitting algorithm for correcting the barrel-pincushion distortion, the proposed scanner delay strategy is described in Section IV, Section V presents the experimental verification, and Section VI concludes the paper.

\section{BARREL AND PINCUSHION DISTORTION}

As shown in Fig. 1, which is the two dimensional laser galvanometer scanning system, the laser beam reflected by two mirrors is focused by F-Theta lens and then projected on to the workbench. While the rotation of mirrors are controlled by motors and the rotation angle is always between -22.5 degrees and 22.5 degrees. Fig. 2 shows the block diagram of galvanometer scanning system. The incident direction of laser beam is parallel to the $\mathrm{x}$ axis. The deflection angles in the directions of $\mathrm{x}$ and $\mathrm{y}$ axes change as the rotation of $x$ mirror and y mirror, which are shown as $\theta_{\mathrm{x}}$ and $\theta_{y}$ in the Fig. 2. The $\theta_{x}$ and $\theta_{y}$ are zero when the laser beam is vertical to the workbench (the $\mathrm{x}-\mathrm{y}$ coordinates) and the projection of laser beam on the workbench is $(0,0)$. When the laser beam irradiate on the workbench, the scanning trajectory can be described as following [10]-[12]:

$$
\left\{\begin{array}{l}
y=d \cdot \tan \theta_{y} \\
x=\left(\sqrt{d^{2}+y^{2}}+e\right) \tan \theta_{\mathrm{x}}
\end{array}\right.
$$

where, $d$ is the distance between coordinate origin and the axis of $y$ mirror, $e$ is the distance of $x$ mirror and y mirror.

From equation 1, the scanning of image is realized actually by the control of $\theta_{x}$ and $\theta_{y}$. It's clear that $\theta_{y}$ relates to the coordinate of $x$ and $y$, while $\theta_{x}$ affects $x$ only. Supposing $\theta_{x}=\theta_{0}$ and $\theta_{0}$ is a constant, the equation 1 can be written as:

$$
\left(\frac{x}{\tan \theta 0}-e\right)^{2}-y^{2}=d^{2}
$$

The equation 2 describes a hyperbola, which means that there is distortion in the direction of the $\mathrm{x}$ axis in the two dimensional galvanometer scanning system. This distortion can be depicted in Fig. 3 and usually is called as pincushion distortion.

The deflection angles of $\theta_{x}$ and $\theta_{y}$ are not simply in linearity with the coordinates of $x$ and $y$ respectively. To simplify the control system, $\tan \theta_{x}$ and $\tan \theta_{y}$ are approximately replaced with $\theta_{x}$ and $\theta_{y}$ respectively. Then, the equation 1 can be written as:

$$
\left\{\begin{array}{l}
y^{\prime}=d \cdot \theta_{y} \\
x^{\prime}=\left(\sqrt{d^{2}+y^{2}}+e\right) \theta_{x}
\end{array}\right.
$$

However, due to $\theta_{x}<\tan \theta_{x}$ and $\theta_{\mathrm{y}}<\tan \theta_{y}$, it is clear that $y^{\prime}<y$ as well as $x^{\prime}<x$, which leads to the barrel distortion in the direction of $x$ axis and $y$ axis, which is shown in Fig. 4(a). The synthetic error caused by barrel and pincushion distortion is shown in Fig. 4(b).

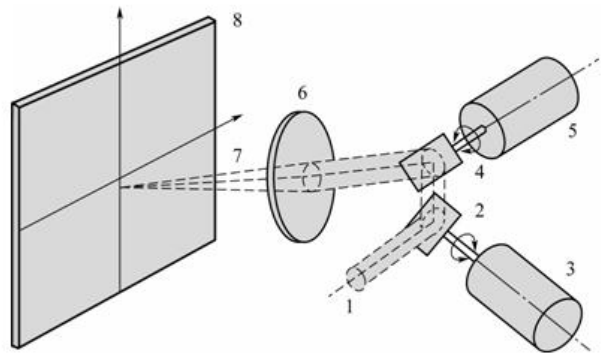

1- laser beam, 2,4- galvanometer mirrors, 3,5- motors, 6- F-Theta lens, 7projection of laser beam on the workbench, 8- workbench Fig. 1. Laser galvanometer scanning system.

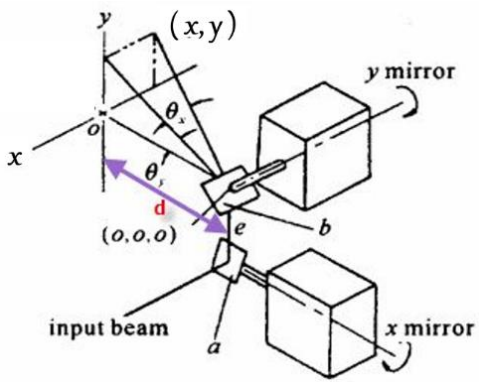

Fig. 2. Block diagram of galvanometer scanning system.

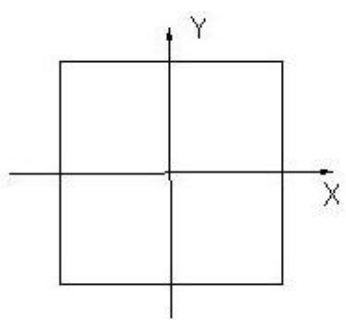

(a)

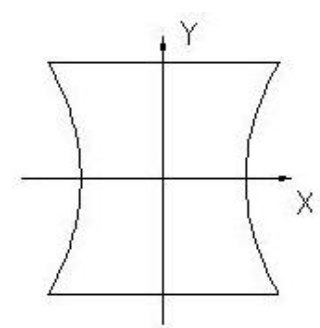

(b)
Fig. 3. (a) Original scanning path, (b) Pincushion distortional scanning path.

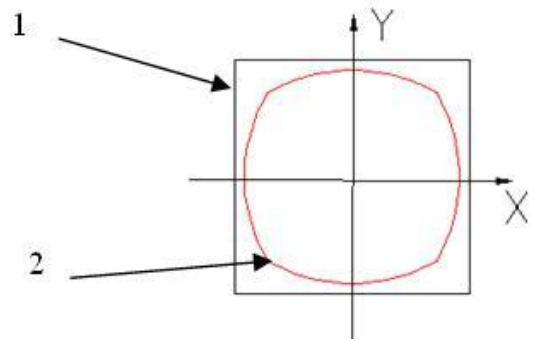

(a)

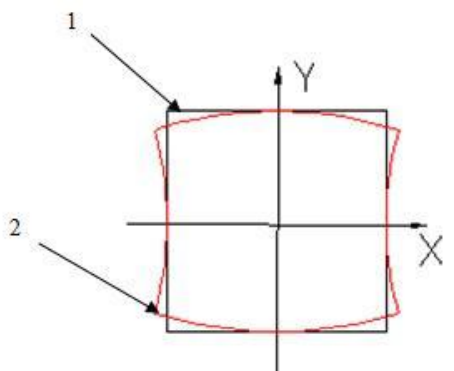

(b)

1-original path, 2-distortional path

Fig. 4. (a) Barrel distortion, (b) Barrel-pincushion distortion.

\section{CONIC FITTING AlgORITHM}

To compensate the error caused by barrel-pincushion 
distortion with conic fitting algorithm has been proposed in some papers [13], [14]. As shown in Fig. 5(a), the distortional path can be fitted with a conic, and the distortion can be calculated. Then a distortion in opposite direction is added to the original path to correct the distortion. From Fig. 5(b), equation of the conic can be written as:

$$
x=y^{2} / p
$$

Then the maximum distortion can be described as Eq.5 and Eq. 6 shows the distortion. The correction equation in the increasing $x$ direction is shown as Eq.7.

$$
\begin{gathered}
\delta_{x \max }=p-y^{2} / p \\
\delta_{x}=\delta_{x \max } \cdot x / p \\
X=k \cdot \delta_{\mathrm{x}}+p
\end{gathered}
$$

where, $\delta_{x \text { max }}$ is the maximum distortion in the increasing direction of $x$ axis, $\delta_{x}$ is the distortion in the increasing direction of $x$ axis, and $\mathrm{k}$ is correction factor.

Furthermore, the correction equations of the negative direction of $x$ axis can be achieved with the same method.

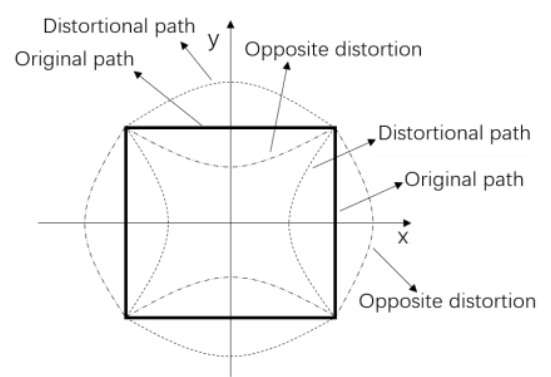

(a)

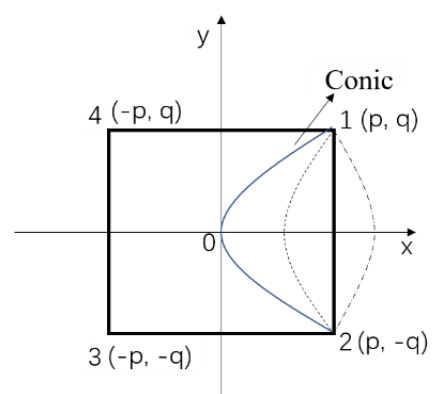

(b)

Fig. 5. (a) The model of correction algorithm, (b) the model of conic algorithm.

\section{SCANNER DELAY STRATEGY}

The non-uniform laser energy deposition in AM process impacts the local material curing characteristics and creates inhomogeneous material property such as stain or stress in the finished parts. Such finished parts may have potential defects and rough surface. Therefore uniform laser density is critical for AM process. In this section, a scanner delay strategy will be discussed to prevent the non-uniform laser power density and the errors which caused by inaccurate scanning movement. The scanner delay strategy includes four kinds of delays, which are laser on-off delay, jump delay, mark delay and Corner delay.

\section{A. Laser on-off Delay}

As shown in Fig. 6, when galvanometer begins to work or stops, the laser will not be opened or turned off immediately but delay for a certain time due to the lower scanning speed. The appropriate laser on delay (LOnD) time and laser off delay (LOffD) time are meaningful to keep uniform laser power density on the material during the beginning and ending phases. Fig. 7 shows effects of the excessive LOnD time and the deficient LOnD time. The deficient LOnD time, which means there will be excessive laser power density to cure material, leads to the excessive accumulation, and the excessive LOnD time could result in contour error. While the excessive and deficient LOffD time cause the excessive and deficient accumulation of material respectively.

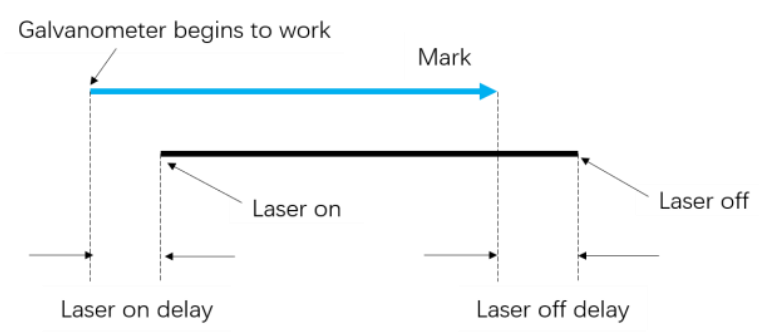

Fig. 6. Laser on-off delay.

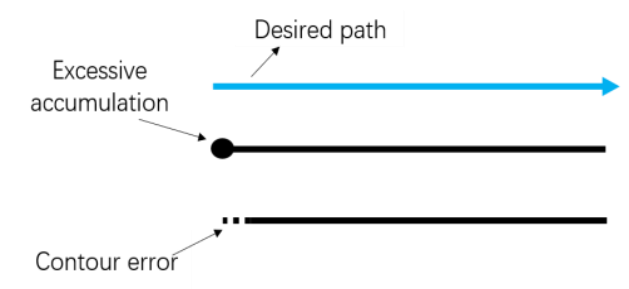

Fig. 7. Effects of the excessive and deficient LOnD time.

\section{B. Jump Delay}

As Fig. 8 shows, the jump delay works when the current scanning path jumps to other paths. The motors are still unstable when the jump ends, which could result in disordered path. The jump delay is designed to avoid this error while the laser will be turned off during the jump and delay for a certain time after the jump ends. However, the excessive jump delay time leads to a lack of material accumulation as well as low efficiency, and the deficient jump delay time leads to excessive accumulation of material.

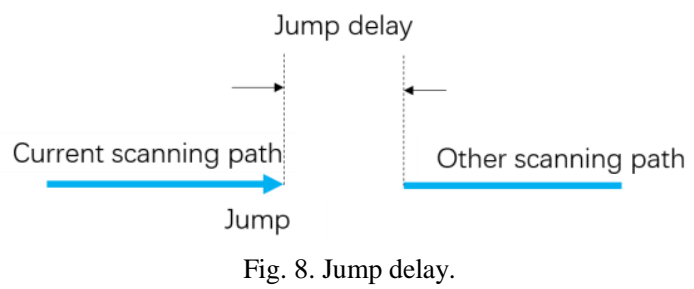

\section{Mark Delay}

The mark delay only works before the command of jump, which is shown in Fig. 9(a), the galvanometer motors and laser will continue to work during the mark delay time. Due to the error between coordinates in command and actual coordinates, the contour error which is shown in Fig. 9(b) could be caused when the jump happens without the mark delay or with the deficient mark delay time. While the excessive mark delay time could result in low efficiency and excessive material accumulation. 


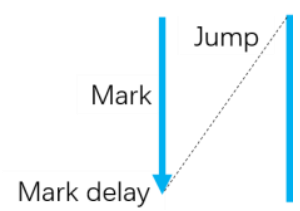

(a)

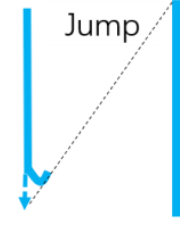

(b)
Fig. 9. (a) Mark delay, (b) contour error caused by the lack of mark delay.

\section{Corner Delay}

Because of the error between coordinates in command and actual coordinates, the contour error could be produced when the scanning path runs into a corner. Fig. 10(a) shows this contour error due to the lack of the corner delay time, while Fig. 10(b) shows the excessive accumulation of material caused by the excessive corner delay time.

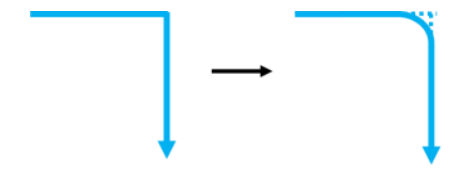

Desired path

Contour error

(a)

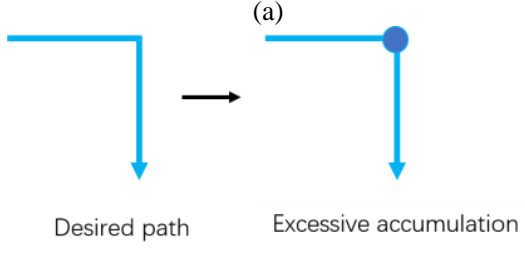

(b)

Fig. 10. (a) Contour error caused by the lack of corner delay, (b) excessive accumulation.

\section{EXPERIMENTAL VERIFICATION}

To validate the performance of conic fitting algorithm and proposed scanner delay strategy, a SLA based 3D printer with galvanometer scanning technology was designed and the control system is shown in Fig. 11, which is implemented on FPGA and STM32. Two models as shown in Fig. 12 were fabricated with the designed 3D printer and compared. The original model was fabricated without any correction algorithm or scanner delay strategy, while the improved one was fabricated with the conic fitting algorithm and the proposed scanner delay strategy.

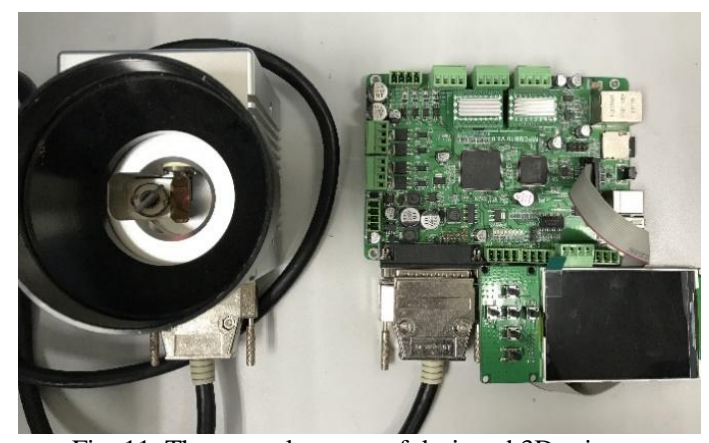

Fig. 11. The control system of designed 3D printer.

The results are shown in Fig. 13. It is clear from Fig. 13(a) that there is a significant distortion in outline of the original part, while it does not happen with the improved one. From Fig. 13(b), it can be seen that the original part has a serious path error when the scanning path runs into a corner and the scanning path of improved one is more accurate. Furthermore, the results also indicate that there is excessive material accumulation on the surface of the original one which leads to the rougher surface than that of the improved one. From the experimental studies, it is proved that the conic fitting algorithm can correct the distortion of galvanometer scanning technology for AM, and the proposed scanner delay strategy is helpful for the galvanometer scanning based AM to achieve the satisfactory precision and better surface finish.

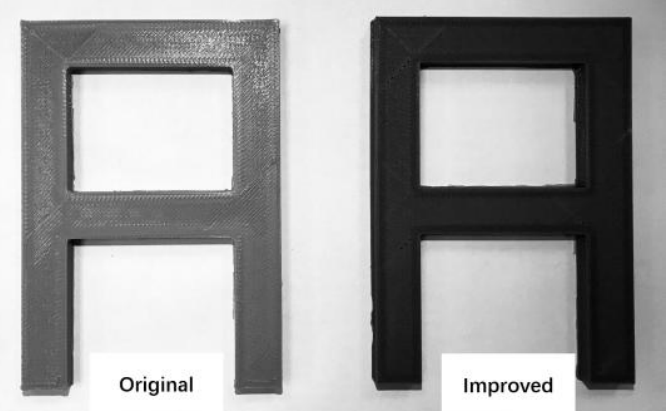

Fig. 12. The model fabricated by designed SLA 3D printer.

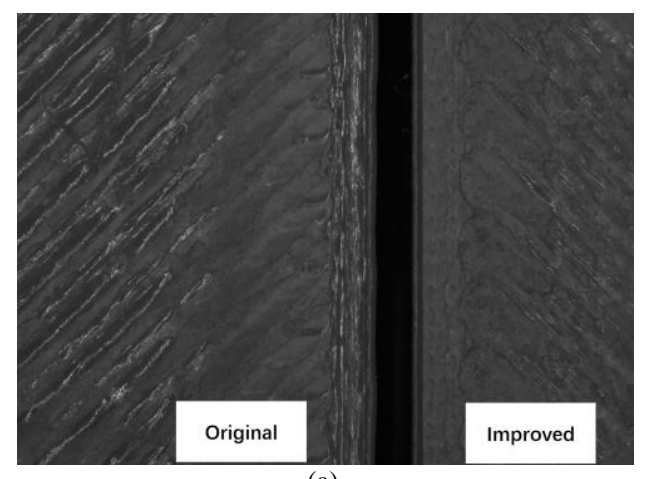

(a)
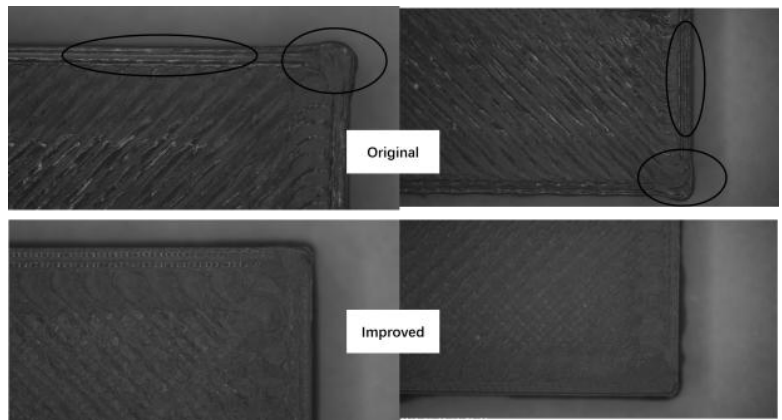

(b)

Fig. 13. (a)Comparison of distortion between original part and improved part, (b) Comparison of path error at corner between original part and improved part.

\section{CONCLUSION}

This paper analyzed the barrel-pincushion distortion of two dimensional galvanometer scanning system, and investigated the performance of conic fitting algorithm for galvanometer scanning based AM to correct the distortion. This paper also proposed a scanner delay strategy to improve the manufacturing precision of AM with galvanometer scanning technology. The experiment was conducted with SLA 3D printing which based on laser galvanometer scanning technology. While the results indicated that the conic fitting algorithm has a contribution to compensate the barrel-pincushion distortion, the scanner 
delay strategy can avoid the excessive material accumulation effectively and correct the error which caused by the inaccurate scanning movement. Therefore, it was easy to reach the conclusion that the conic fitting algorithm and the proposed scanner delay can improve the geometric precision as well as surface finish of the parts fabricated with galvanometer scanning based AM. As the conic fitting algorithm and the proposed scanner delay strategy are easily to be implemented, the work of this paper is meaningful for the application of laser galvanometer scanning technology with $3 \mathrm{D}$ printing.

\section{ACKNOWLEDGEMENTS}

This paper is supported by the National Natural Science Foundation of China (General Program, NO.51175356).

\section{REFERENCES}

[1] B. P. Conner, G. P. Manogharan, A. N. Martof et al., "Making sense of 3-D printing: Creating a map of additive manufacturing products and services," Additive Manufacturing, vol. 1, no. 4, pp. 64-76, 2014.

[2] K. V. Wong and A. Hernandez, "A review of additive manufacturing," International Scholarly Research Network (ISRN) Mechanical Engineering, 2012.

[3] D. Faidel, A. Laskin, W. Behr, and Natour, "Improvement of selective laser melting by beam shaping and minimized thermally induced effects in optical systems," in Proc. 9th International Conference on Photonic Technologies, 2016.

[4] Q. Zhao, C. Wang, and J. H. Yang, "Graphic distortion analysis and correction arithmetic research on laser galvanometer scanning," Journal of Changchun University of Science and Technology, vol. 35, no. 4, pp. 63-75, 2012.

[5] C. K. Zhu and L. P. Ding. "Research on forming qualities during selective laser melting based on scanner delay strategy," Applied Laser, vol. 37, no. 2, pp. 207-212, 2017.

[6] X. Luo, J. Li, and M. Lucas, "Galvanometer scanning technology for laser additive manufacturing," Proceedings of SPIE, Vol. 10095, 2017.

[7] G. Y. Li, Y. Z. Wang, J. R. Han, and S. Y. Liu, "Geometry algorithm of galvanometer system correction for rapid prototyping of machine parts," Machine Tool \& Hydraulics, vol. 42, no. 19, pp. 28-30, 2014.

[8] Q. Zhao, C. Wang, and J. H. Yang, "Graphic distortion analysis and correction arithmetic research on laser galvanometer scanning," Journal of Changchun University of Science and Technology, vol. 35, no. 4 , pp. 63-65. 2012.

[9] H. W. Yoo, S. Ito, and G. Schitter, "High speed of laser scanning microscopy by iterative learning control of a galvanometer scanner," Control Engineer Practice, vol. 50, pp. 12-21, 2016.
[10] H. P. Jia, Y. S. Shi and J. Xie, "Error analysis of laser galvanometer scan system and its geometrical correction arithmetic," Opto-Electronic Engineering, vol. 34, no. 8, pp. 37-40, 2007.

[11] Y. Zhao and B.-H. Lu, "Pillow shaped distortion correction algorithm of galvanometric scanning system," Chinese Journal of Lasers, vol. 30, no. 3, pp. 216-218, 2003 .

[12] Y. J. Gong, F. Xia and J. X. Sun, "Geometric distortion correction for laser marking system," Electronic Measurement Technology, vol. 37 no. 10, pp. 62-65, 2014.

[13] Z. Chen and X. D. Liu, "Calibartion arithmetic of the quick software for laser galvanometer scanning system," J. Huangzhong Univ. of Sci. \& Tech., vol. 31, no. 5, pp. 68-69, 2003.

[14] T. L. Wang, H. F. Gao, and D, C. Wang, Distortion Analysis and Algorithm Research on Laser Galvanometer Scanning, no. 7, pp. $19-22,2009$

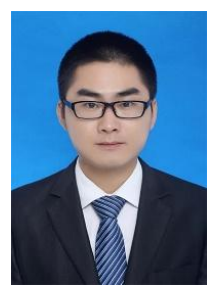

Huang Jigang is a $\mathrm{PhD}$ in School of Manufacturing Science and Engineering from Sichuan University. His current research interests are in the area of 3D printing and Mechatronic.

Qin Qin is a PhD in School of Manufacturing Science and Engineering from Sichuan University. His current research interests are in the area of 3D printing and Automatic controls.

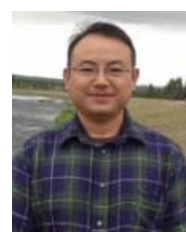

Fang Hui is a professor in mechanical engineering in Sichuan University. He is the associate dean of School of Manufacturing Science and Engineering in Sichuan University. His current research focused on 3D printing, $\mathrm{CNC}$ and precision manufacturing technology.

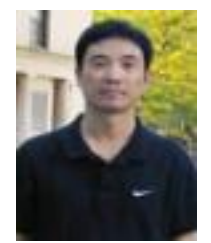

Wang Jie is a professor in mechanical engineering inSichuan University. He is the dean of School of Manufacturing Science and Engineering in Sichuan University. His current research focused on CAD/CAM, Design and manufacturing. 